\title{
Laryngeal Papillomatosis
}

National Cancer Institute

\section{Source}

National Cancer Institute. Laryngeal Papillomatosis. NCI Thesaurus. Code C157733.

Recurrent respiratory papillomatosis that affects the larynx. 